\title{
Cultura familiar y patrones reiterativos en salud sexual y reproductiva de madres adolescentes en la frontera colombovenezolana (departamento Norte de Santander y Táchira)*
}

Vivian Vanessa Arenas Villamizar1; Marlen Karina Fernández Delgado²; María Carolina Martínez Santana ${ }^{3}$

* Capítulo de libro producto de los proyectos de investigación: 1) Análisis comparativo de la Maternidad adolescente en la frontera Norte de Santander - Táchira. Fecha de inicio: enero de 2017. Fecha de finalización: diciembre de 2017. Universidad Simón Bolívar - sede Cúcuta - Colombia. 2) El Táchira en Cifras 2014. Maternidad y embarazo en adolescentes. Universidad Católica del Táchira, Venezuela. Fecha de inicio: 01 de enero de 2014 Fecha de finalización: diciembre de 2017.

1 Psicóloga egresada de la Universidad de la Sabana, especialista en Ciencias de la Familia y magíster en Mediación Familiar de la Universidad de los Andes de Santiago de Chile. Docente investigador de la Universidad Simón Bolívar, Cúcuta, Colombia. Correo: v.arenas@unisimonbolivar.edu.co

2 Psicóloga egresada de la Universidad de Pamplona, especialista en Psicología Clínica y de la Salud de la universidad Autónoma de Bucaramanga y Magistra en Psicología de la Salud de Pontificia Universidad Javeriana de Cali. Docente investigador de la Universidad Simón Bolívar, Cúcuta, Colombia. Correo:m.fernandez@unisimonbolivar.edu.co

3 Psicóloga egresada de la Universidad Santo Tomás de Aquino, especialista en Gerencia Social de la Universidad Simón Bolívar con maestría en Intervención Social de la Universidad Internacional de la Rioja, España.

Correo:mmartinez@unisimonbolivar.edu.co 


\section{RESUMEN}

El objeto de este capítulo es describir la cultura familiar y los patrones reiterativos en salud sexual y reproductiva de las madres adolescentes en la frontera colombovenezolana. Para el desarrollo de los pilares básicos que determinan este escrito se desarrollaron dos proyectos independientes de investigación enmarcados en un convenio de cooperación institucional: "El Táchira en Cifras, 2014, Maternidad y Embarazo en adolescentes" ejecutado por el Observatorio Social de la Universidad Católica del Táchira (Venezuela) y "Maternidad de las adolescentes en Norte de Santander" elaborado por la Universidad Simón Bolívar sede Cúcuta (Colombia). Los dos estudios se hicieron desde un enfoque de Investigación cuantitativo con diseño no experimental de alcance descriptivo y corte transversal. Se usó un muestreo aleatorio estratificado por afijación proporcional en Norte de Santander y Táchira. Se aplicó una encuesta a una muestra de 406 personas en Norte de Santander y 235 adolescentes en el estado Táchira entre 10 y 19 años. Los resultados indican que la familia juega un papel fundamental en la configuración de creencias, abordaje de la sexualidad y en la adquisición posterior de información. Por otra parte, las circunstancias de las madres de las adolescentes con respecto a la edad del embarazo tienden a repetirse intergeneracionalmente convirtiéndose en un patrón reiterativo. Respecto a las relaciones familiares y la forma como se determinan, se pudo concluir que en este estudio no es factor contingente pues antes del embarazo las adolescentes tenían una relación cercana con sus familias y consideraban que sus padres estaban atentos de sus necesidades.

Palabras clave: embarazo adolescente, cultura familiar, patrones reiterativos, salud sexual y reproductiva.

\section{FAMILY CULTURE AND REPETITIVE PATTERNS IN SEXUAL REPRODUCTIVE HEALTH OF ADOLESCENT MOTHERS IN THE BORDER BETWEEN COLOMBIA AND VENEZUELA (DEPARTMENT OF NORTE DE SANTANDER AND TACHIRA)}

\section{ABSTRACT}

The purpose of this chapter is to describe the family culture and repetitive patterns 
in sexual reproductive of adolescent mothers in the border between Colombia and Venezuela. For the development of the basic pillars that determine this writing developed two independent research projects framed in an agreement of institutional cooperation: "The Tachira in figures, 2014, maternity and pregnancy in adolescents" executed by the social observatory of the Catholic University of Tachira (Venezuela) and "Maternity in adolescents from Norte de Santander" elaborated by the university of Simon Bolivar headquarters of Cucuta (Colombia). The two studies were made from a quantitative research approach with a non-experimental design of descriptive scope and cross-section. A stratified unsystematic sampling was used for proportional sampling in Norte de Santander and Tachira. A survey was applied to a sample of 406 people from Norte De Santander and 235 adolescents in the state Tachira between 10 and 19 years of age. The results indicate that the family plays a fundamental role in the configuration of beliefs, approach to sexuality and in the subsequent acquisition of information. On the other hand, the circumstances of mothers of adolescents with respect to age to pregnancy have to be repeated intergenerationally, becoming a repetitive pattern. In respect to family relationships and how they were determined it was possible to conclude that this study is not a contingent factor because before adolescence adolescents had a close relationship with their relatives and considered their parents were aware of their needs.

Keywords: adolescent pregnancy, family culture, repetitive patterns, sexual reproductive health.

\section{INTRODUCCIÓN}

El Fondo de Población de las Naciones Unidas (UNFPA, 2013) refiere que frente al embarazo a temprana edad en el mundo, 20.000 adolescentes dan a luz todos los días; 70.000 muertes son registradas en esta población anualmente por complicaciones del embarazo y parto y se dan 3,2 millones de interrupciones inseguras del embarazo entre adolescentes.

En Colombia se detectó - a partir de 1990- que el embarazo adolescente estaba aumentando sistemáticamente, alcanzando su mayor índice en el 
2005 e iniciando su descenso a partir de 2010 como lo referencia Profamilia en la ENDS (2015).

Los datos anteriores reiteran que esta es un problemática de salud pública y social que tiene una importante incidencia en la familia, tal como lo afirman Noguera y Alvarado (2012), quienes expresan que el embarazo a temprana edad se establece como un problema homogéneo y de afectaciones negativas a nivel social y de salud para los adolescentes y su núcleo familiar, no solo por los efectos colaterales que genera en la salud de las madres y sus hijos, como en las consecuencias que se dan al obligar a los jóvenes a apropiarse de un rol prematuro de adultos, donde no se tienen las capacidades físicas, emocionales, ni sociales para asumir la maternidad de manera autónoma y responsable, pues ellos no han consolidado su propia identidad, ni han definido un proyecto de vida viable, tampoco cuentan con relaciones significativas, ni la búsqueda de autonomía, ni de vinculación (Acción Social, 2010).

Rojas, Méndez y Álvarez (2016), citando a Fernández y Escalona (2011), explican que como problema homogéneo, el embarazo en la adolescencia es una patología del ciclo vital, que tiene su inicio en los procesos educativos, comportamentales y de contexto socio-cultural, comprendida como un constructo que se intenta imponer como límites de rango de edad biológica de la mujer, entre los 12 y los 17 años de edad.

Estos mismos autores plantean que actualmente el embarazo a temprana edad es una anomalía en el ciclo vital, desde el punto de vista médico, psicológico y además para el grupo familiar en el que se desarrolla la adolescente. De igual manera, proponen que la maternidad se debiera realizar en condiciones ideales como que se dé en una edad adulta, que haya una constitución de familias nuevas, adecuados antecedentes genéticos y de crianza, al igual que buenas condiciones económicas para el crecimiento y desarrollo de los hijos. 
Es por esto que Castrillón (2007) define la familia como el sistema organizativo de mínimo peligro para la crianza y el mejor facilitador de protección de la trasmisión cultural, de apropiados hábitos sanitarios, que incluyen los de la sexualidad y la reproducción.

El concepto de familia ha cambiado drásticamente porque sus funciones se han transformado; por mucho tiempo su papel principal consistía en transmitir el patrimonio, económico y moral de una generación a otra pero hoy en día la construcción de la identidad personal es la prioridad en la familia tanto en las relaciones conyugales como en las relaciones que se dan entre padres e hijos. Tomando como base un movimiento de psicologización creciente y la sentimentalización del fenómeno familiar hoy en día, dominan la intersubjetividad que es su atributo principal, al igual que el amor es su principio de funcionamiento (Théry 1997 citado por Gracia y Musito, 2000).

Jones et al., 1995 y Alberdi, 1995 (citados por Gracia y Musitu, 2000) dicen que:

Para la mayoría de la población, la cualidad esencial de la vida familiar es un acuerdo o un compromiso emocional. Las "buenas familias" se supone que proporcionan intimidad (proximidad, relaciones satisfactorias), promueven la educación de los hijos y la escolarización, potencian el bienestar material de sus miembros, su salud física y mental y su autoestima. (p.53)

Lo que propone este capítulo es describir la cultura familiar y los patrones reiterativos en salud sexual reproductiva de madres adolescentes en la frontera colombovenezolana (departamento Norte de Santander y Táchira).

Para su desarrollo se consideró abordar los siguientes temas: en la primera sección se expondrá brevemente el concepto de embarazo adolescente, después se explicará la familia como sistema; la tercera tratará las funcio- 
nes familiares y la última detallará la cultura familiar y patrones reiterativos en el embarazo de las adolescentes basados en los resultados de las investigaciones preliminares.

\section{EMBARAZO ADOLESCENTE}

El embarazo en la adolescencia se define por la OMS (2011), como uno de los principales factores que contribuyen a la mortalidad materna. A nivel psicológico trae consigo un impacto en la estructura mental, que tiene como resultado el fracaso en la adquisición de independencia, logro de la propia identidad y preparación para la vida adulta (Noguera y Alvarado, 2011). También se desencadenan estados de depresión durante el embarazo y en el postparto (Ministerio de Salud y Protección Social, 2014). A esto se le suman las condiciones del individuo las cuales co-existen a partir de los factores emocionales y culturales que se ven representados desde el valor que los adolescentes le asignan a la condición de ser madre o padre.

Fernández y Soto (2017) retoman la descripción de la UNICEF (2014) quienes plantean que entre los factores asociados al embarazo adolescente y sus efectos se encuentran las características del hogar de la adolescente: los ingresos de sus progenitores, sus niveles de educación y la condición de pobreza del hogar. Pero también hay otros contextuales relevantes, como el acceso a una educación sexual integral, a los distintos métodos de planificación familiar y, en general, a la garantía del ejercicio de sus derechos.

A nivel emocional, existen dos miradas con respecto a la maternidad, la primera de ellas hace referencia a cómo el adolescente mantiene socialmente la idealización de la maternidad-paternidad como condición necesaria para la existencia, y a nivel cultural-individual estos determinantes están muy relacionados con la construcción de género, pues la maternidad y la paternidad tienen un valor social y cultural representativamente fuerte y se instaura en el individuo desde edades muy tempranas, y por 
consiguiente, se traduce en realidad para edades menores en relación con las edades socialmente permitidas para ser madre, pues lo importante es cumplir con el rol asignado y esperado de la reproducción, sin tener en cuenta la edad o el ciclo de vida en el que se encuentre (Salinas, Castro y Fernández, 2014).

Y la segunda mirada, la cual para algunos tiene una visión negativa, se asocia a la reproducción de la pobreza, en donde a largo plazo puede afectar, pero a mediano plazo termina viabilizando proyectos de vida, pues el embarazo se torna fuente de motivación, que trae a la vida de estas madres felicidad y amor, así como sentido de vida según los autores Komura (2008), Nieto et al. (2011) y Noblega (2009).

Así mismo, para Mottrie, Coster y Duret (2006), algunas adolescentes consideran el embarazo como el inicio hacia otro tipo de relaciones filiales; el tener un hijo es el paso a la adultez y por tanto la obtención del poder que se encontraba en los padres y al que ellas no tenían acceso por su condición de adolescente. Es decir, que toman posición dentro de su linaje, permitiéndoles así la construcción de una identidad sexual adulta, lo que se complementa con la percepción que algunas adolescentes tienen de esta condición, cuando refieren que les permite alcanzar una posición importante dentro de la sociedad.

Dado que el embarazo en la adolescencia es un problema de salud pública cuyas causas son multifactoriales, se retoma lo expuesto en el CONPES 147 acerca de la teoría de los determinantes sociales conceptualizada como el conjunto de variables individuales, sociales y estructurales que, relacionadas entre sí, condicionan el proceso vital y explican el conjunto de fenómenos asociados a la salud sexual y reproductiva de adolescentes y jóvenes.

Gallo y Molina (2008) describen cómo los determinantes se refieren a di- 
ferentes aspectos de los individuos y las sociedades que pueden incidir o condicionar los resultados en diferentes problemáticas y que requieren la atención e intervención de múltiples actores. En el enfoque de determinantes sociales de la Organización Mundial de la Salud (OMS), en el 2011 se define a los determinantes como los factores sociales, políticos, económicos, ambientales y culturales que condicionan el proceso vital, y específicamente el proceso salud-enfermedad. La acción de los determinantes, a diferencia de los factores de riesgo, tiene diferentes efectos y jerarquías sobre una sociedad, y su intervención está interrelacionada. El proceso de determinación social de la vida, de la salud y la enfermedad, combina de manera específica para cada comunidad, efectos estructurales del marco económico y político, así como factores relacionados con las condiciones laborales, ambientales y de organización social, y finalmente determinantes proximales, relacionados con las prácticas individuales y familiares.

ELCONPES 147 hace una descripción acerca de los determinantes próximos, intermedios y distales los cuales constituyen una explicación de los aspectos asociados al embarazo adolescentes. Los determinantes próximos están relacionados con las características individuales en los que se destacan factores biológicos como el desarrollo puberal y factores del comportamiento como el inicio de relaciones sexuales, la nupcialidad o las uniones tempranas, el uso de los métodos de anticoncepción, y el acceso a servicios de salud y educación (Zamudio y Rubiano, 1999 citado en Florez, 2000). En particular tiene gran incidencia la edad de inicio de las relaciones sexuales y el momento en que se dan las uniones o la nupcialidad. Así mismo, se encuentra la configuración de proyectos de vida propios, las prácticas de género, las creencias del individuo y sus comportamientos alrededor de la sexualidad, las relaciones sexuales, la maternidad y la paternidad; son, entre otras, algunas de las variables que pueden incidir en las decisiones sobre la vida sexual y reproductiva de las y los adolescentes.

Así mismo, los determinantes intermedios se refieren a la familia o el 
hogar en el que se encuentre el niño, niña o adolescente, y en su escuela, entre los que se consideran las condiciones familiares, la existencia de abuso o violencia, la supervisión y el diálogo entre padres o cuidadores y adolescentes y jóvenes, las normas de funcionamiento del hogar, el cuidado por mantener al niño, niña o adolescente en el sistema escolar, la formación de calidad en Salud Sexual y Reproductiva y Derechos Sexuales Reproductivos (DSR) en la escuela, entre otros. En especial se considera el nivel educativo como un factor protector, no solo por las oportunidades que representa tener una mayor educación, sino por las habilidades que se adquieren para favorecer el propio desarrollo; tener percepciones más ajustadas y positivas sobre sí mismo, sobre la identidad de género, y por la capacidad que se adquiere para valorar y evaluar la adecuación de las normas sociales, las creencias sobre el rol de género, el lugar que se ocupa en las familias, el trabajo, la comunidad o la sociedad, e incluso sobre los comportamientos de autocuidado, lo que conduce a tener comportamientos sexuales menos riesgosos y decisiones con mayor autonomía y responsabilidad.

De igual manera, los determinantes distales están relacionados con los ingresos, pobreza, la cobertura y acceso oportuno a servicios públicos, la oportunidad de participar en las decisiones públicas y el ejercicio de la democracia, los legados culturales que trascienden las instituciones, las comunidades, el macroentorno social, las normas sociales sobre la sexualidad, la feminidad, la masculinidad, las relaciones de pareja o la participación de las niñas(os) y adolescentes en los procesos de decisión e identidad social, de la existencia de oportunidades de desarrollo personal y alternativas de vida y la atención de la comunidad a los DSR, a la prevención de su vulneración y la adecuada valoración de los mensajes transmitidos a través de las Tecnologías de la Información y la Comunicación (TIC), así como en aspectos estructurales como la pobreza o la permanencia escolar y la existencia de opciones para la generación de ingresos. 


\section{CONCEPTO DE FAMILIA COMO SISTEMA}

Desde la década de los años 60, uno de los enfoques más útiles para el estudio de la familia es la teoría general de los sistemas que considera a un sistema como un conjunto de elementos en interacción dinámica; cada uno cumple una función con respecto al todo pero no se reduce a la individualidad de sus partes y sus funciones, son más que la simple suma de ellas. Por esta razón, la familia es un sistema compuesto por un conjunto de personas que se interrelacionan entre sí y son interdependientes, es decir, que lo que le pasa a uno afecta a los demás, y viceversa (Xitlali y Rivas, 2011).

El concepto de familia ha variado de múltiples formas y desde puntos de vistas diferentes, esto hace pensar en lla como un sistema que permite verla como un todo y no a partir de sus partes de manera aisladas, no relacionadas; se le considera entonces como un organismo relacionado en forma dinámica con sus subsistemas o atributos.

Se identifican de manera generalizada las contribuciones de Ludwig von Bertalanffy, biólogo, y del matemático Norbert Wiener como las personas más influyentes en el origen y desarrollo de lo que se conoce como la Teoría General de los Sistemas. Tal como lo indica Paley (1997, citado por Gracia y Musitu, 2000), el acento que hace Bertalanffy (1974), radica en la comprensión de las propiedades de las totalidades, lo cual indicaba que era un esfuerzo de la ciencia clásica de aislar los elementos del universo observable con la esperanza de que al reunir esos elementos de nuevo el todo sería inteligible. Sin embargo, para Bertalanffy era necesario comprender no solo los elementos de un sistema sino, de ello lo más importante, sus interrelaciones. De esta manera, la idea fundamental de la teoría de sistemas será que debe ser entendido como una totalidad y no puede comprenderse, examinando sus partes individuales en aislamiento. Esto quiere decir que el todo es mayor que la suma de sus partes. 
En esta misma fundamentación, Smith (1995, citado por Gracia y Musitu, 2000) propone que una familia se puede conceptualizar como un sistema porque posee las siguientes características:

a) los miembros de una familia se consideran partes interdependientes de una totalidad más amplia; la conducta de cada miembro de la familia afecta a todos los otros miembros de la familia; b) para adaptarse, los sistemas humanos incorporan información, toman decisiones acerca de las distintas alternativas, tratan de responder, obtener feedback acerca de su éxito y modificar la conducta si es necesario; c) las familias tienen límites permeables que las distinguen de otros grupos sociales; y d) al igual que otras organizaciones sociales, las familias deben cumplir ciertas tareas para sobrevivir, tales como el mantenimiento físico y económico, la reproducción de miembros de la familia (nuevos nacimientos o adopción), socialización de los roles familiares y laborales, y el cuidado emocional. (Smith, 1995, citado por Gracia y Musitu, 2000, p.140)

Barroso (1997 citado por Gracia y Mutisu, 2005) define la familia como un sistema que se organiza de diferentes maneras, se compone de varios miembros importantes, cada uno con necesidades, capacidades, contextos y objetivos individuales, que interactúan buscando continuamente la integración y su bienestar. Cabe destacar que lo más importante no son los elementos individuales sino la interacción del todo lo que hace que se dé la unión, dándole sentido y dirección. También se determina como una red invisible que se estructura de tantos elementos como miembros que la componen, con una energía de crecimiento que los mantiene interconectados en un contacto permanente y les permite dar apoyo a todos sus miembros, proporcionando tomar conciencia de la importancia de su participación que es la sensibilidad de la red.

Broderick (1993, citado por López y Escudero, 2003) refiere que la familia es un sistema abierto, donde se promueve constantemente la búsqueda de 
metas y la autorregulación. Lo que la diferencia de otros sistemas sociales y la hace única, es que su estructura esté determinada por la edad y el género. Esta se modela por sus "características estructurales (tamaño, complejidad, composición, estadio vital), las características psicobiológicas de sus miembros (edad, sexo, fertilidad, salud, temperamento, etc.) y posición sociocultural e histórica en el ambiente" (p.23).

Este autor también propone que la familia se diferencia por el criterio de pertinencia en el que se incluyen dos características: la estructural, en la que se plantea que las familias son exclusivas en la estructuración de los géneros y las generaciones porque no todas las familias incluyen pareja, ni todas son heterosexuales, a pesar de que culturalmente se espera que la pareja sea activa sexual y reproductivamente. Además, no todas las familias contienen más de una generación, sin embargo la generación más joven es el nexo biológico (o cuando se da la adopción legal) de la generación mayor.

La siguiente característica diferencial tiene que ver con la forma de las relaciones, que pueden ser difusas cuando contienen gran cantidad de situaciones; en las particularistas se dan reglas que dependen de las relaciones que se establecen entre ellos, por ejemplo, se espera que el esposo se comporte de forma diferente con su esposa con respecto a otra mujer; afectivas porque es apropiada la expresión de sentimientos en un amplio rango y adscritas porque no se pertenece a la familia por elección y su estatus es independiente de acciones voluntarias como las que son definidas por el género o parentesco (Broderick, 1993, citado por López y Escudero, 2003).

García (2008) refiere que la familia como sistema tiene unas características esenciales: hace parte de un suprasistema o sistema mayor (la familia extensa o familia de origen y la sociedad) aplicando el principio sistémico de recursividad, que establece que lo que se aplica al sistema, también lo hace para el subsistema y el suprasistema; los subsistemas que componen 
la familia son el conyugal, fraterno y familia extensa; establece unas fronteras o límites entre los subsistemas que varían siendo rígidos, flexibles y permeables; las relaciones que se forman con los hijos se fundamentan en los roles complementarios y se basan gran tiempo en la dependencia; tiene una estructura es decir, sus relaciones son más o menos estables, esenciales y permanentes. Además está en proceso de transformación continua, lo que alude a su dinámica; la familia transita una serie de etapas de desarrollo denominado ciclo vital familiar; se espera que la familia y sus miembros cumplan diversas funciones, algunas de ellas en interrelación con otros sistemas como las tareas de cuidado; la familia como sistema para regular su funcionamiento define roles y establece reglas para cada miembro.

Complementando la definición de la familia desde la perspectiva sistémica Vasco, Bermúdez, Escobedo, Negret y León (1999, citados por García, 2008), consideran otras características:

La totalidad que se origina de la relación entre los sistemas y subsistemas que la componen se puede analizar, es decir, permite hacer cortes en sus diferentes aspectos en un momento específico como al evaluar la convivencia; admite hacer modelos, mapas mentales y representaciones, lo que significa que es moldeable. La familia está orientada a metas, tiene propósitos y se rige por su propio proceso, indicando que es autoorganizada.

Narez (2009) explica que la teoría general de los sistemas apoya la descripción de la familia como un sistema sociocultural que incluye el concepto de adaptabilidad, puesto que en todas se presenta una compleja dinámica que preside sus patrones de convivencia y funcionamiento. Si esta dinámica es funcional, es decir, adecuada y flexible, aportará a la armonía familiar y al desarrollo sólido de los sentimientos de identidad, seguridad y bienestar de sus miembros.

A manera general, la definición de familia en la teoría de sistemas está 
concebida como un sistema social cuya característica principal es la de un sistema abierto, dinámico, autorregulado y dirigido hacia la consecución de metas, y más allá de esto, con unas configuraciones propias estructurales (complejidad, tamaño, estadio vital) y unas características psicobiológicas de cada uno de sus miembros (edad, género, salud, temperamento, etc.) y una posición sociocultural e histórica en su entorno más amplio. Por lo anterior, las familias no pueden definirse en términos estáticos o estructurales, debido a que sus partes se encuentran relacionadas de manera dinámica entre sí y con el entorno. Esto demanda focalizarse en los procesos y en los patrones de esos procesos en el tiempo. En la interacción de sus partes, surgen las cualidades de la familia, por lo tanto son emergentes.

La organización en patrones recurrentes y repetitivos que pueden ser observados, se configura en otra cualidad de las familias como sistemas abiertos y dinámicos. De los patrones observables se pueden deducir las reglas que gobiernan el sistema y que se estructuran jerárquicamente. Las normas forman parte de las reglas, estas permiten mantener y regular las relaciones entre los integrantes del sistema, y mantener y regular las relaciones con el entorno. Los sistemas más amplios están interconectados con los sistemas familiares; estos incluyen una red de relaciones familiares más extensa, el vecindario, las escuelas, el ámbito laboral; de igual manera los suprasistemas o los sistemas más amplios también incluyen lo cultural, racial, lo étnico o regional (Gracia y Musitu, 2000).

Como cualquier otro sistema, la organización es muy importante en la familia; a diferencia de los otros sistemas, se da una estrecha relación y dependencia de sus miembros. Pero la eficacia de la organización depende del grado de satisfacción de sus objetivos, esto es, de los objetivos de sus integrantes y los de la sociedad. Con el fin de lograr sus objetivos a través de su organización, la familia debe asegurar y conseguir energía tanto de las fuentes internas como de las externas. Sus integrantes aportan al sistema familiar energía o importan energía para sus propósitos particulares (Anderson y Carter, 1994). 
El límite de la familia lo conforma todo lo que determina la conducta y se manifiesta en la intensidad y frecuencia de la interacción entre sus integrantes. La intensidad respecto a los intercambios afectivos es su principal característica; esto de alguna manera incluye que alguno de sus componentes participe en una apretada red de sentimientos que pueden ser tanto positivos como negativos, sin sentir algún tipo de restricción al hacerlo porque es un espacio en el cual transmite la libertad de ser uno mismo.

En la medida en que los miembros de la familia vienen y van, parientes, amigos íntimos, vecinos e hijos adoptivos, pueden ser incorporados dentro de los límites de una familia determinada. Los límites son a su vez físicos y psicológicos, y si ambos aspectos no coinciden, el resultado puede ser en extremo angustiante; es el caso, de un hijo que no se encuentra, pero su madre lo considera aún presente. En este sentido "se ha descubierto que la ambigüedad del límite está íntimamente relacionado con la tensión y la disfunción total de la familia" (Pasley y Ihinger-Tallman, 1989 citados por Anderson y Carter, 1994, p.232).

La sociedad defiende estos límites dándole importancia a la familia en aquellas ocasiones en los que afloran esos sentimientos profundos, tales como funerales, bodas y fiestas religiosas, el día de la madre y el día del padre son celebraciones creadas para reforzar los sentimientos e intereses familiares. La familia como sistema social, requiere realizar intercambios más allá de sus límites; algunas familias llegan a la entropía debido a los escasos intercambios afectivos con el medio.

Otros conceptos aplicables a las familias son el de diferenciación y la especialización. Su especialización es la de satisfacer la necesidad de protección y afecto de sus miembros. Según Anderson y Carter (1994) la reducción de sus funciones es el resultado de la diferenciación dentro de la sociedad moderna. En el seno de la familia, la diferenciación y la especialización se reflejan en la distribución de los roles; es así como en la satisfacción de las

\section{3}

Vivian Vanessa Arenas Villamizar; Marlen Karina Fernández Delgado; María Carolina Martínez Santana 
expectativas sociales, algunos de los miembros de la familia se dedican a ganar el sustento con la aprobación de los demás. Las expectativas del rol están determinadas por la edad, el sexo y se estructuran en cada sistema familiar.

Ahora bien, los roles parentales se determinan como fundamentales en el desarrollo de los integrantes de la familia, tanto para el crecimiento del propio padre o madre como para el de los hijos. Estudios señalan que los roles son intercambiables y sugieren que los niños tienen un mejor desarrollo; cuando hay consistencia, existe una relación de cuidado de los hijos, por parte de alguno de los dos padres. El óptimo resultado en los niños se podría asociar con un conjunto de conductas parentales tales como la demostración de cariño, responsabilidad ante las necesidades de los niños, la estimulación, la asistencia diaria y la supervisión a través de una disciplina no coercitiva, sirviendo de modelos de conducta positiva. Existe una complementariedad entre el rol de padre y madre, culturalmente el padre personifica la autoridad y la madre el afecto (Roizblatt, 2009).

Para Anderson y Carter (1994)" toda conducta familiar está influida por el estilo y la eficacia de la comunicación" (p.236); esto quiere decir que se realiza una transferencia de símbolos y significados orales y gestuales, y que de igual manera hay una transferencia de energía que se requiere para alcanzar los objetivos propuestos por el sistema. Cada familia posee un estilo de comunicación que le es propio. Estas pautas características operan dentro de los límites de la familia e interactúan con los sistemas más externos; ese intercambio contribuye a la supervivencia de la identidad del sistema (Maturana y Varela, 1998). Es así como una familia en particular posee una combinación única de pautas de comunicación que influye de manera poderosa en la conducta de sus integrantes; de ellos se deriva que las pautas de comunicación son indispensables para comprender a la familia debido a la importancia e intensidad de los sentimientos que se ponen en juego en el intercambio. La comunicación en la familia 
es considerablemente sutil y compleja debido a las diferentes funciones que cumple la familia. En el intercambio de energía, se pueden transmitir muchos significados que no sean compatibles.

La adaptación es una función de la familia; por ende el sistema familiar es un sistema de ajuste a los cambios sociales. Le ha correspondido sobrevivir a la familia en situaciones de guerra, de revolución industrial y tecnológica y a los cambios sociales que vuelven obsoletas las pautas tradicionales de imitación, debido a que posee fundamentalmente la capacidad de modificar su estructura y sus funciones a fin de adaptarse a los profundos cambios del entorno. Gracias al intercambio de energía de sus componentes e intercambio de energía con los sistemas significativos del entorno es que la familia logra mantenerse. Una familia se adapta mal cuando se le dificulta acomodarse a los exigentes cambios de las demandas de sus miembros y del entorno. En la rigidez (morfostasis) y en la dedicación mayor de la energía al mantenimiento de las estructuras, no será capaz de manejar los requerimientos externos ni el desarrollo individual de sus integrantes, por lo tanto solo mantiene sus funciones básicas y en consecuencia es disfuncional. Y si permanece de manera permanente en un estado de inestabilidad y transición, no proporciona la estabilidad que sus integrantes requieren para reparar las heridas del yo y para ser simplemente ellos mismos en una atmósfera de intimidad afectiva.

Entre las destacadas contribuciones a la aproximación sistémica de la familia se encuentra la de David Olson (1982, citado en López y Escudero, 2003) quien ha conjugado su aproximación terapéutica con la investigación científica rigurosa. Su modelo, el Circunflejo se estructura a partir de dos dimensiones: la cohesión y la adaptabilidad. La cohesión está configurada por los lazos emocionales que los miembros de la familia tienen unos con otros y el grado de autonomía personal que experimentan. De otra manera, la adaptabilidad es la habilidad del sistema familiar para cambiar sus estructuras de poder, relaciones de roles, y reglas de relación en respuesta

\section{5}

Vivian Vanessa Arenas Villamizar; Marlen Karina Fernández Delgado; María Carolina Martínez Santana 
a una situación de estrés y evolutivo. Para este autor, las familias funcionales serían entonces aquellas cuyos valores de cohesión y adaptabilidad se mantienen en unos valores medios; de no ser así, la familia interpreta que hay problemas en sus relaciones. La contribución sistémica de Olson (1982), tiene gran aplicación dentro de la familia, aun desde las primeras relaciones de pareja hasta las etapas de la vejez y se ha posicionado como uno de los mejores ejemplos de fusión entre la ciencia y la terapia. De igual manera es una de las bases conceptuales y aplicadas del Sistema de Evaluación Familiar (López y Escudero, 2003 p.69).

\section{FUNCIONES FAMILIARES}

La conformación actual de la familia en sus diversos tipos, identifica cambios y adaptaciones de ciertas funciones. Es así, como aquellas que estaban en exclusiva competencia de la familia como el cuidado de enfermos, de niños, ancianos, ha pasado a entidades del Estado; esto reafirma el proceso de constante adaptación que atraviesa la familia como una institución esencialmente dinámica. Si bien, la comprensión de esos cambios y ajustes de la familia podemos enmarcarlos en unas tendencias de la vida moderna, En este sentido, Gracia y Musitu (2000) advierten sobre sus causas en un incremento de los divorcios, la incorporación de la mujer al mundo laboral, el incremento de las familias monoparentales, la cohabitación como forma de las parejas de convivencia, conformación de familias homosexuales, etc., representando cambios dramáticos en las formas familiares e incidiendo en el declive de la familia tradicional sin que ella sea suplida por un nuevo tipo "ideal" de familia.

Describir las funciones de la familia es reconocer las tareas que la sociedad misma le ha asignado; podemos entonces decir que una de las formas de identificar a la familia es por las funciones que ellas desempeñan. Asegurar la supervivencia de los seres humanos es la responsabilidad que históricamente le ha sido asignada. 
García (2008) relaciona las funciones que como grupo se espera que la familia proporcione a sus integrantes: asigne un nombre a los descendientes, legitimando la paternidad que se hace a través del apellido; el cuidado de los niños, asegurando su subsistencia en el cumplimiento de sus necesidades básicas de alimento, abrigo, educación y protección física; proveer un ambiente que respalde el apoyo emocional, la armonía pero también la diferencia; facilitar la identidad personal, la individualización y la construcción de su identidad en la convivencia; promover el apoyo mutuo mediante la asignación de tareas en armonía con las edades de sus integrantes; promover el sentido de pertenencia partiendo de la unión y la solidaridad; facilitar niveles de seguridad para desarrollar la autonomía acorde a las edades; transferir los valores y normas; propiciar el apoyo y soporte emocional a sus integrantes en momentos de adversidad; preparar a sus miembros al ejercicio de la vida pública en un escenario de debate democrático. Todas estas funciones hay que entenderlas en un contexto histórico y cultural, ya que a partir de ello, se establecen diferencias de acuerdo al sexo, es decir como hombres y mujeres y como padres y madres.

Esta relación de funciones puede resumirse en: función reproductora, protectora, socializadora y económica. Sobre la función reproductora es por la tradición judeocristiana, la primera tarea asignada a la familia y este aspecto es el que determina su conformación. No obstante, como ya se ha mencionado, por las tendencias modernas se hace referencia a distintas opciones de familia, aunque persiste la utilización del término de familia para referirse a tener hijos.

Contar con una familia amplia de descendientes era un imperativo, ya que facilitaba el trabajo en el campo en el caso de las familias de escasos recursos y más hijos para garantizar la continuidad del apellido y el ejercicio del poder en las clases privilegiadas. La situación hoy en día es diferente y es así cómo las presiones económicas cobran relevancia asi como los aspectos psicológicos. Se ejerce una presión de familiares y allegados para tener

\section{7}

Vivian Vanessa Arenas Villamizar; Marlen Karina Fernández Delgado; María Carolina Martínez Santana 
los hijos. Respecto a los aspectos psicológicos, se conserva la esperanza de que el hijo renueve el matrimonio que pueda estar pasando por tiempos de crisis.

El impacto de las políticas natales se vieron modificadas por el auge de los anticonceptivos, lo que determinó que las mujeres hicieran una clara separación en el ejercicio de la sexualidad de los aspectos reproductivos de los placenteros, disociación que realizan los hombres por el hecho de que no se embarazan y por las representaciones de la sexualidad. De igual manera, como consecuencia de los anticonceptivos la maternidad como un proyecto femenino, pasó a ser planeada y aplazada.

La función protectora de la familia se refiere a cómo es asumida por los integrantes de la familia y cómo esta función ha ido cambiando a lo largo del tiempo; sin embargo, no puede verse esta función de manera aislada a las condiciones de cada país, desde su marco social y político pues estos aspectos la afectan en su realidad económica y de bienestar. En un contexto histórico, los abandonados y desprotegidos eran concentrados en lugares como hospicios y conventos. Otra era la situación de las clases altas, en las que la atención y cuidado directo era dado por las nodrizas pero, en tanto que el Estado evoluciona, son sus instituciones las que surgen para apoyar a la familia, especialmente en lo tocante a los derechos y su imperativo restablecimiento.

La familia desarrolla también una función socializadora, es decir, proporciona la internalización de normas y valores de una cultura y transmite pautas que les permite a sus integrantes relacionarse con los demás y vivir en sociedad. La escuela es clave en la función socializadora, al igual que los medios de comunicación.

Finalmente, las funciones económicas de las familias permiten proveerla de los elementos necesarios en la subsistencia y satisfacción de sus nece- 
sidades relacionadas con la salud, educación, vivienda, etc.; pero como ya se ha apuntado, hay una relación de la economía con el desarrollo de las funciones familiares: "las funciones son numerosas de acuerdo al nivel económico, así también se proporcionan de modo diferente en las distintas clases" (García, 2008, p.198).

Otros autores le otorgan a la familia distintas funciones que son muy importantes para su estabilidad "El grupo familiar se constituye como agregado de ocio y consumo, de plataforma de ubicación social, de núcleo de relación social, de palanca para la constitución de patrimonio, de cauce para hallar empleo, de punto de apoyo y de recurso de amparo en caso de crisis y de unidad de prestación de cuidados asistenciales y de salud" (Flaquer, 1998, citado por Gracia y Musitu, 2000, p.53).

Ahora bien, en una opinión en consenso se podría decir que la mayoría de las personas afirman que la cualidad esencial de la vida familiar es un acuerdo o compromiso emocional. Para Jones et al., (1995, citados por Gracia y Musitu, 2000), las "buenas familias" proveen intimidad (proximidad, relaciones satisfactorias), promueven la educación de los hijos, potencian el bienestar material de sus integrantes, su salud física y mental al igual que su autoestima. De igual manera se infiere que existen en las familias tareas esenciales a las que se enfrentan todas aquellas personas que viven en grupo, entre ellos el cuidado de los niños, la regulación de la sexualidad, el establecimiento de un sentimiento de identidad y los límites, modelos de intimidad como una pareja y como alguna forma de unidad familiar, negociando roles en términos de divisiones, de obligaciones y toma de decisiones y definiendo algunas reglas sobre los modelos de obligaciones o deberes mutuos.

Lo que define a una familia entonces puede considerarse que es la negociación y la complementariedad de estas tareas. Esto sugiere una concepción de la dinámica de la vida familiar como un proceso. Son los intentos 
continuos de solucionar esas tareas que personifican o expresan la vida familiar más que la forma particular-nuclear, uniparental, reconstituida, extensa, comuna, etc.; lo que emerge como un intento de solución que las personas pueden y se les permite intentar se construyen culturalmente, pero tal modelo dinámico nos libera de la trampa de tratar de definir cualquier forma de vida familiar como la familia.

\section{RESULTADOS}

La función educativa es una de las principales que cumple la familia, a través de ella se facilita y acceden a conocimientos y habilidades que proporcionan nuevos aprendizajes. De igual manera, la función social es imprescindible pues la interacción en este sistema es el primer proceso de socialización del individuo que va a facilitar en las siguientes etapas de su evolución la adquisición de una serie de conductas de adaptación que le van a servir a sus integrantes en posteriores periodos de su vida.

En ese proceso de socialización se presentan diversos escenarios, situaciones y factores del orden psicosocial que pueden resultar problemáticos para la población adolescente, sus familias y grupo social; entre ellas, se encuentra lo referente al reconocimiento, abordaje y manejo individual y colectivo de los conocimientos, actitudes y prácticas sobre sexualidad. Además se pueden incluir otras dificultades tales como el aperturar conversaciones en el tema, posturas relacionadas con el inicio de relaciones sexuales a temprana edad, los tópicos frente al tema del embarazo y las infecciones de transmisión sexual (Jones, 2010).

González, Leal, Molina y Chacón (2013) aducen que las circunstancia de las madres adolescentes con respecto a la edad del primer embarazo tienden a repetirse por lo cual la edad del primer embarazo se convierte en un patrón reiterativo, que puede tener consecuencias aún más graves en la 
vida de sus hijas, quienes suelen tener dificultades escolares y se embarazan a una edad incluso más temprana que sus propias madres.

En los estudios realizados, una de las preguntas estuvo centrada en identificar la edad en la que las madres de las participantes habían tenido su primer hijo, debido a que existe una fuerte asociación entre embarazo adolescente en una generación anterior con el embarazo adolescente en la siguiente, es decir, es muy probable que una mujer que fue madre adolescente sea hija de una mujer que también fue madre adolescente, lo que hace que sea un patrón reiterativo. En los resultados del departamento Norte de Santander se encontró que la edad mínima es de 13 años, la máxima es de 32 y la media se encuentra en los 18,9 años. En el Táchira los resultados son similares en la edad mínima, la máxima se encuentra en 33 y la media en 18,4 años. Lo anterior indica que en las dos fronteras, teniendo en cuenta la edad en la que las madres de las adolescentes quedaron embarazadas por primera vez existe una coincidencia en la edad mínima y no se evidencia una diferencia significativa en la media y en la edad máxima; sin embargo comparado con las jóvenes en Norte de Santander y Táchira en las que coincide la edad mínima de embarazo a los 11 años y en la edad máxima de 19, se convierte en un patrón reiterativo; la media en Norte de Santander es de 16 años y en el Táchira 17,3; de lo que se infiere que en las hijas la edad mínima es dos años menos cuando se presentó su primer embarazo y con respecto a la edad máxima existe una diferencia significativa.

Retomando la función bilógica de la familia, la cual la define como un grupo humano cuya razón de ser es la procreación, la crianza y la educación de los hijos, complementada con la dimensión psicológica que destaca la importancia de la familia en el mundo actual porque de ella depende la fijación de las aspiraciones, valores y motivaciones de los individuos, resulta responsable en gran medida de su estabilidad emocional, tanto en la infancia como en la vida adulta. Es así como la familia tiene la habilidad 
de proporcionar un lugar para el apoyo emocional y para las relaciones complementarias y satisfactorias (Gracia y Musitu, 2000).

En consecuencia, la aceptación parental (calidez e involucramiento) promueve el establecimiento efectivo de normas y una buena comunicación; reduce la probabilidad que los adolescentes se unan a grupos de influencias negativas y asuman conductas sexuales de riesgo. La conducta y actitudes de los padres y adultos frente a las relaciones sexuales influyen significativamente en el desarrollo de sus hijos a través de un ambiente armonioso y la información explícita sobre la sexualidad, lo cual puede constituirse como un factor protector en la medida que exista cercanía, orientación y comunicación con sus padres o adultos.

Las relaciones entre los padres, madres e hijos es un elemento decisivo como función protectora, puesto que permite un proceso de desarrollo integral que repercute positivamente en los hijos, afianzando niveles de autonomía que les facilitan el hacerse cargo de su propia vida. Con respecto a la importancia de la relación familiar se retoman los resultados obtenidos para el departamento Norte de Santander que indagó sobre la relación con la familia de origen antes del embarazo; se encontró que el 49,3\% correspondiente a 200 adolescentes describen que su relación familiar antes del embarazo fue buena; el 28,3\% (115) regular; el 12,6\% (51) muy buena; el 7,1 \% (29) mala y el 2,5 \% (10) como muy mala. En la población del Táchira el 62,5 \% (147) refieren que fue buena; el 23,1 \% (54) muy buena; el 10,8 \% (25) la describen como mala y el 3,7\% (9) como muy mala. Se infiere que los porcentajes más altos tanto en Norte de Santander como en el Táchira corresponden a buena relación de las adolescentes con sus familias de origen antes del embarazo; otro hallazgo importante es que en el Táchira no se evidencian relaciones intermedias, es decir son muy buenas, buenas, o muy malas o malas.

Teniendo en cuenta la descripción de la relación con la figura paterna, los 
resultados obtenidos para Norte de Santander indican que el 37,4 \% (152) fue cercana; el 23,4 \% (95) lejana; el 15,5\% (63) muy cercana; el 14,3\% (58) afirma no tuvo figura paterna y el 9,4 \% (38) argumenta que fue muy lejana. Para el caso del Táchira el 39,9 \% (94) afirma que fue cercana; en porcentaje correspondiente al $17 \%$ (40) se encuentra la relación muy cercana y lejana, seguido del 15,4\% (36) quienes manifiestan no tuvieron figura paterna y el 10,7 \% (25) muy lejana. Lo anterior indica que coinciden en las participantes de las dos fronteras los porcentajes más altos respecto a la buena relación con su figura paterna.

Londoño (1994, citado en Acción Social, 2010), indica que diversos estudios realizados con la población adolescente muestran que existe una probabilidad de presentarse una gestación cuando se suman factores biológicos, socioculturales dentro de los que se incluye el uso inadecuado del tiempo libre, la necesidad de asumir roles de adultos, sentimientos de soledad, desafecto, baja autoestima, los enamoramientos románticos e irracionales, todo ello unido a la falta de educación de los padres. La percepción que tienen los hijos acerca de lo que sus padres esperan de ellos, la deficiente comunicación y la falta de interés de los padres a las necesidades de sus hijos constituyen factores determinantes frente a la construcción de relaciones afectivas positivas y la capacidad de tomar decisiones autónomas responsables y planeadas que ayuden a sus hijos a tener criterios claros a la hora de iniciar su vida sexual.

En los estudios, se buscó conocer la percepción que las adolescentes tenían acerca de si el padre o representante se interesaba por ellas y las personas con las que se relacionaba y por las actividades que hacía diariamente; los resultados para Norte de Santander evidencian que el 33,5 \% (136) refiere que siempre hubo supervisión y acompañamiento; el 32.3\% (131) casi siempre; el 27,1 \% (110) pocas veces y el 6,7\% (27) nunca. En los hallazgos del Táchira se evidencia que el 60,1\% (141) siempre; el 18,5\% (43) como casi siempre; el 15,1\% (35) pocas veces y el 6,3\% (15) nunca. 
Debido a que la supervisión o monitoreo parental es un factor decisivo que no solo pone límites a sus comportamientos sino a la vez se establece contacto con los amigos para conocerlos, se pude inferir que de los dos estudios existe mayor acompañamiento y supervisión de los padres en el Táchira que en Norte de Santander.

Con relación al embarazo adolescente, este se ha considerado mayoritariamente una consecuencia previsible del aumento de las relaciones coitales prematrimoniales entre adolescentes; a partir de estas situaciones surgen cuestionamientos acerca de si se debe permitir o prohibir y si la aceptación que los padres dan a los noviazgos a temprana edad constituye un factor de riesgo para el embarazo adolescente. En el estudio realizado para Norte de Santander se encontró que la edad que tenían las adolescentes al momento de la aceptación del novio se encuentra como mínima a los 11 años, máxima 19 y la edad media 15 años; en el caso del Táchira la edad mínima es de 10 años, la máxima 19 y la media 14,93. En cuanto a las reglas familiares en las dos fronteras hay una similitud que se refleja en la aceptación de la primera relación de sus hijas en edades tempranas.

La sexualidad es un tabú en los contextos estudiados. Los adultos hablan poco de sexualidad y lo hacen menos sobre sexualidad adolescente, al punto que el solo hecho de hablar sobre el tema, se considera un acto de provocación o un detonante que puede desencadenar actos sexuales. En los estudios se ha podido identificar que el tabú y el miedo se transfieren de generación en generación como patrón reiterativo a través del silencio y la brecha de comunicación que se reproduce entre padres/madres e hijos/as. Si bien las limitaciones de la comunicación intergeneracional son varias y de carácter general; existen algunos factores particulares que impiden hablar en torno a la sexualidad (UNICEF, 2014).

Piñeros (2014), refiere que los padres no comunican este tema porque en ocasiones no esperan que las adolescentes inicien las relaciones sexuales a temprana edad, por lo cual no han considerado relevante darles esta infor- 
mación, así mismo Sevilla y Orcasita (2014) describen que el proceso de reflexión en torno a las propias experiencias de vida de padres y madres, está marcado por el silencio y la falta de comunicación con adultos o cuidadores.

De manera recurrente, hombres y mujeres señalan que el tema era en muchas ocasiones prohibido o abordado de manera indirecta y reactiva, es decir, solo abordado ante la insistencia de ellos que como adolescentes, eventualmente se atrevían a preguntar sobre todo lo relacionado con los cambios propios de la pubertad. En los resultados de las investigaciones, se encontró frente a la pregunta cómo había sido la educación sexual por parte de la familia antes del embarazo, en Norte de Santander al 45,8 \% (186) nunca le hablaron sobre el tema; el 26,6 \% (108) quería que no se embarazara para que terminara sus estudios; así mismo se encuentra que al $17,2 \%$ (70) se le decía que tuviese cuidado con los hombres que engañan, embarazan y se van; en menor proporción con un 3,9 \% (16) se encuentran aquellas que consideraron que habían recibido una buena educación sexual y el 6,4\% (26) que no saben o no responden. Con respecto a los resultados del Táchira se encontró que al 36 \% (85) nunca le hablaron sobre el tema; el 22,2 \% (5) refiere que su familia quería que no se embarazara para que terminara sus estudios; al $16 \%$ (38) le decían que tuviese cuidado con los hombres que engañan, embarazan y se van y el 25,8 \% (61) consideraron que recibieron una buena educación sexual. De lo anterior se deriva que el mayor porcentaje en Táchira y Norte de Santander -según las jóvenes- no recibieron de sus familias una preparación en los tópicos de la educación sexual. No obstante, se presenta una divergencia en el Táchira porque la prevalencia indica que las adolescentes tienen la percepción de haber recibido una buena orientación sobre sexualidad.

El intervalo protogenésico se refiere al período entre la unión de la pareja y el nacimiento del primer hijo. En la ENDS (2015) lo describen como un indicador importante para entender el contexto de nacimiento y crianza 
de los hijos y la relación con la constitución de uniones, ya sean legales o consensuales. Respecto a los nacimientos en el marco de las uniones, en la encuesta publicada en el 2015 se evidenció que el $12 \%$ de los nacimientos ocurre en mujeres nunca unidas; $21,7 \%$ ocurre antes de la fecha de la primera unión y un $17 \%$ ocurre en los primeros 8 meses desde la primera unión; lo que indica que un 33,7 \% de los nacimientos se dieron fuera de una unión y un 50,7\% de los nacimientos se concibieron antes de una unión. Con respecto a los resultados de la investigación, para Norte de Santander se encontró lo siguiente: el 26,1% (57) se casó o unió antes de quedar embarazada por primera vez; el 53,7 \% (117), se casó o unió después de quedar embarazada por primera vez; el $1.4 \%$ (3) se casó o unió después de su segundo o posterior embarazo; el 3,2 \% (7) se casó y después tuvo su primer embarazo, y el 15,6 \% (34) no responde. Estos resultados coinciden con lo expuesto en la ENDS (2015) que refiere que aunque en Colombia las relaciones sexuales previas a la primera unión son cada vez más comunes, la unión (legal o consensual) continúa siendo la institución predominante para la tenencia y crianza de los hijos. En los resultados del Táchira se evidenció que el 55,5 \% (90) se casó o unió antes de quedar embarazada por primera vez, el 40,1 \% (65) se casó o unió después de quedar embarazada por primera vez y el 4,4 \% (7) se casó o unió después de su segundo o posterior embarazo. De lo anterior se puede deducir que en el Táchira hay una mayor tendencia a formalizar las relaciones después del embarazo en comparación con Norte de Santander, favoreciendo el cuidado del niño por parte de los dos progenitores, lo que fortalece la función de protección de la familia.

Frente al significado e importancia que las adolescentes le atribuyen a la conformación de una "familia", la relacionan con la confirmación de su identidad, el logro del reconocimiento y la aceptación social con la que ganan estatus y evidencian madurez. En cuanto a la postura asumida por la familia ante la unión o casamiento, en el departamento Norte de Santander se encontró que el 23,4 \% (51) decidió que se uniera o se casara; el 15,6 \% (34) 
apoyó materialmente la unión o casamiento (ofreciendo vivienda, enseres, alimentación); el 36,2 \% (79) lo permitió con normalidad, no hubo oposición; el 10,1\% (22) se opuso pero igual se casaron o unieron, y el 14,7\% (32) no respondió la pregunta. Con respecto a los resultados del Táchira el 6,2\% (10) decidió que se uniera o se casara; el 33,2 \% (54) apoyó materialmente la unión o casamiento (ofreciendo vivienda, enseres, alimentación); para el $41,2 \%$ (67) se permitió con normalidad, no hubo oposición, y finalmente el 19,4 \% (31) refiere que su familia se opuso pero igual se casó o unió. Los resultados reflejan que respecto a las dos fronteras se manifiesta un aspecto social inherente al cuidado que tienen las familias de su imagen como grupo social, considerando como relevante la formalización de la unión de sus hijas y el apoyo en recursos para su mayor sostenimiento.

El asumir nuevos roles maternos y paternos en los adolescentes, puede llegar a desencadenar frecuentes conflictos debido a que en esta nueva condición contravienen sus propios intereses, necesidades y planes futuros, implicando cambios en sus proyectos de vida, que no solo obstaculizan sus estudios sino su relación familiar y de pareja, debido a que cuando asumen la constitución de un nuevo hogar no son conscientes de las consecuencias, compromisos de carácter social, legal, económico y afectivo.

En muchas ocasiones los adolescentes asumen la convivencia o contraen matrimonio bajo presión como una forma de asumir los efectos del embarazo no estando preparados, porque tienen poca capacidad para tomar decisiones y no cuentan con la madurez y con los recursos necesarios para asumir las responsabilidades de los mayores. Frecuentemente se evidencia que la unión se dio por influencia de familiares o amigos y bajo la orientación de ellos. De acuerdo a lo anteriormente planteado se pretendió conocer las razones por las que la familia decidió, apoyó o permitió que se unieran o casaran. En las respuestas dadas por las adolescentes de Norte de Santander se encontró que el 13,8 \% (30) lo hizo para evitar problemas dentro de la misma familia; el 4,1 \% (9) para evitar que la familia sufriera 
daños de parte de su pareja; el 22,9 \% (50); para que tuviera una buena pareja y mejorara su situación; el 16,1 \% (35) para que no fuera una madre soltera; el 16,1 \% (35) por costumbre, pues consideran que es normal que se haga así; el $9 \%$ (2) refirió otras razones dentro de las que se encuentra que nunca la apoyaron y para que ambos se responsabilizaran, y un 26,1 \% (57) no responde. En las participantes del Táchira el 7,8 \% (10) refirió que fue para evitar problemas dentro de la misma familia; el 29,4\% (38) para que tuviera una buena pareja y mejorara su situación; el 13,3 \% (17) para que no fuera una madre soltera; el 31,3\% (41) refirió que fue por costumbre, es normal que se haga así, y 18,3 \% (24) otros, sin especificar. La prevalencia indica que para la familia otra de las razones más importantes para apoyar la unión o matrimonio de sus hijas es el deseo de que ellas tengan mejores condiciones emocionales y económicas y en el Táchira se destaca más que el motivo sea por tradición.

Así mismo, se encuentra que la falta de conocimiento, la escasa supervisión de los padres, la pobre percepción del riesgo, la norma social percibida y las creencias acerca de la sexualidad desencadenan el temprano inicio de las relaciones sexuales, algunas de ellas sin el uso de métodos de anticoncepción que terminan en embarazos no planeados y a temprana edad, estableciéndose relaciones de pareja de convivencia sin que exista conocimiento real del otro; todo se hace basado en la intención de ofrecerle a su hijo mejores condiciones de vida o con la finalidad de asumir su responsabilidad. Esas relaciones ocurren a temprana edad y en muchas ocasiones su nueva condición es producto de una relación pasajera de poco vínculo con su pareja.

Otra condición de riesgo la describe la ENDS (2015) respecto a la diferencia de edad de la mujer con el padre del hijo, reflejando condiciones de asimetría de poder de negociación con su compañero sobre temas de sexualidad, toma de decisiones, planes de vida, etc.; a mayor edad del hombre con respecto a la de la mujer, mayor la asimetría de poder desfavorable para 
ella y mayor la desigualdad de género. Se indagó acerca de la edad que tenían ellas y su pareja cuando se casaron o se unieron por primera vez. En Norte de Santander se encontró que la edad mínima para las mujeres corresponde a 12 años, para los hombres 14 años; la edad máxima para las mujeres corresponde a 19, en los hombres 34; la media 16,2 años para las mujeres y 20,7 para hombres. En el Táchira se encontró que la edad mínima para mujeres es de 13 años, para hombres 12 años; la edad máxima en mujeres fue 19 años, hombres 55 años y la media en mujeres corresponde a 16,18 y en hombres 20,23 años. Los datos demuestran que en el Táchira hay una diferencia significativa en la edad máxima de la pareja de las adolescentes, determinando mayor riesgo de problemáticas sociales como la desigualdad de género.

El temor al abandono de la pareja, la familia o la sociedad en general es uno de los miedos más importantes que acompaña al embarazo adolescente. Por otro lado, la familia juega un rol preponderante en las decisiones e implicaciones del embarazo; por ejemplo, en la continuidad de los estudios, el apoyo necesario no se circunscribe a cuestiones prácticas para viabilizar la asistencia a la escuela, sino también en aspectos emocionales, de orientación, de confianza y empoderamiento (UNICEF, 2014). A partir de ello se indagó en el estudio la importancia que se le da a la aprobación o apoyo de su familia a la hora de tomar decisiones, ante lo cual las adolescentes de Norte de Santander respondieron que es muy importante el 29,8 \% (65); es importante 43,6 \% (95); le es indiferente al 11,9\% (26) y no responde $14,7 \%$ (32). De lo anterior se infiere que la familia, desde su diversidad, sigue siendo la más importante instancia de apoyo que tienen las adolescentes embarazadas y madres. Muchas de ellas terminan aceptando la situación, incluso frente a circunstancias difíciles y acogen a la adolescente, a su bebé y en algunos casos a su pareja. Cuando se alude a "la familia" se hace referencia, por lo general, a un contexto de cobijo, protección y apoyo para la adolescente embarazada. 


\section{METODOLOGÍA EMPLEADA}

La construcción del capítulo "Cultura familiar y patrones reiterativos en salud sexual y reproductiva de madres adolescentes en la frontera colombovenezolana" (departamento Norte de Santander y Táchira) retoma fuentes valiosas y diversas focalizadas principalmente en dos proyectos de investigación "El Táchira en cifras, 2014, maternidad y embarazo en adolescentes" de la Universidad Católica del Táchira (Venezuela) y "Maternidad en adolescentes en Norte de Santander realizada por la Universidad Simón Bolívar sede Cúcuta (Colombia); ambas investigaciones desarrolladas a partir de un convenio de cooperación institucional. Estos estudios, se consolidan como insumos preliminares del proyecto Análisis Comparativo de la maternidad adolescente en la frontera Norte de Santander-Táchira con fecha de inicio del 16 de enero del 2016 con una proyección a terminar en diciembre de 2017.

La estrategia metodológica de estos estudios se fundamenta en los datos positivos de la metodología cuantitativa, no experimental y transversal. A partir de la población determinada para cada contexto, se definió un muestreo probabilístico. Las poblaciones estaban conformadas por madres adolescentes con hijos no menores a 18 meses, tanto del sector rural como del urbano; en Norte de Santander se ubicaron 6.435 madres adolescentes y del Táchira, un total de 1.963. La muestra se definió a través de un muestreo aleatorio simple en poblaciones finitas.

Como técnica de investigación para la recolección de datos se aplicó una encuesta estructurada en Norte de Santander a 406 adolescentes y a 235 adolescentes del Estado Táchira. Se determinó una estratificación con los grupos etáreos de 10 a 14 años, 15 a 17 años, 18 a 19 años.

Se extractan resultados sobre sexualidad y fecundidad a partir de la aplicación de la encuesta, en un análisis comparativo de variables tales como la edad de la primera menstruación, conocimiento del ciclo menstrual y periodo fértil, edad de la primera relación sexual, edad de la pareja cuando 
tuvo la primera relación sexual, edad del primer embarazo, uso del condón durante la primera relación sexual, el primer embarazo corresponde al primer hombre con el cual tuvo la primera relación sexual, tiempo que transcurrió desde las primeras citas, enamoramiento, primeras relaciones sexuales y primer embarazo; y para el análisis comparativo se emplea el análisis univariado de datos (Magallanes et al., 2014; Mazuera et al., 2017).

\section{CONCLUSIONES}

La familia otorga a los individuos las características genéticas, afectivas, educativas y materiales, por lo que se define como un grupo social fundamental de la sociedad (Xitlali y Rivas, 2011). Es por esto que el tipo de relaciones que se construyen entre sus miembros son de gran importancia, dependiendo del grado de satisfacción, de las necesidades biológicas psicosociales y sociales de sus integrantes, por lo que es un sistema que varía en forma permanente; tiene que aprender y desaprender siendo imprescindibles los contactos o conexiones interpersonales que dentro de ellas se dan por conseguir relaciones exitosas y se estimule la interacción saludable fuera de ella (Sauceda 2003, citado por Aigaje y Suárez, 2013).

Se puede suponer que las adolescentes que enfrentan un embarazo a temprana edad, no obtuvieron información o acceso a métodos de planificación y si lo obtuvieron este no fue dado de manera amplia y suficiente, generando baja eficacia a la hora de adoptar comportamientos preventivos, ya que sus intenciones estaban dirigidas a ser madres adolescentes y en casos donde la decisión está tomada, no importa cuánto conocimiento sobre el tema se tenga, las adolescentes referían que a pesar de su edad con sus parejas ya habían hablado del tema y habían decidido formar una familia y estar en otro ambiente, viviendo aparte e independientes. En Táchira hay una mayor tendencia a formalizar las relaciones después del embarazo en comparación con Norte de Santander, favoreciendo el cuidado y protección del niño por parte de los dos progenitores, lo que fortalece la función de protección de la familia. Además, la prevalencia indica que para la familia 
una de las razones más importantes para apoyar la unión o matrimonio de sus hijas es el deseo de que ellas tengan mejores condiciones emocionales y económicas y en el Táchira se destaca que el motivo se da por tradición es decir, la necesidad de cumplir una formalidad religiosa y social.

Sin embargo, es de gran interés que el mayor porcentaje en las dos fronteras las jóvenes no recibieron de sus familias una preparación en los tópicos de la educación sexual, evidenciándose como un patrón reiterativo intergeneracional el no abordar el tema de la sexualidad por ser un tabú.

Se infiere, que en Norte de Santander y Táchira las adolescentes tenían buena relación con sus familias de origen antes del embarazo, confirmado por los resultados que coinciden en las participantes de las dos fronteras respecto a la buena relación con su figura paterna, no siendo en estos casos un factor contingente para el embarazo adolescente pero sí, un elemento decisivo como función protectora que facilita un desarrollo integral que incide positivamente en las hijas, afianzando sus niveles de autonomía y promoviendo en ellas el hacerse cargo de su propia vida.

Otro aspecto importante está relacionado con si se debe permitir o prohibir los noviazgos a temprana edad y si la aceptación de los padres, constituye un factor de riesgo para el embarazo adolescente. En Norte de Santander se encontró que la edad que tenían las adolescentes al momento de la aceptación del novio se encuentra como mínima a los 11 años y en el Táchira a los 10 años, coincidiendo con la edad mínima en la que las adolescentes quedaron embarazadas por primera vez.

En este capítulo se puede concluir que la edad de las madres en su primer embarazo respecto a la edad de sus hijas tienden a repetirse y aun en estas últimas, en edades un poco más tempranas, por lo cual la edad del primer embarazo se convierte en un patrón reiterativo como lo refieren González, Leal, Molina y Chacón (2013) citando que puede tener consecuencias más graves en la generación más joven, porque se embarazan a una edad más temprana que sus propias madres; entre estas dificultades se mencionan las escolares. 
Las familias se repiten a sí mismas, lo que sucede en una generación a menudo se repetirá en la siguiente porque las pautas vinculares en generaciones previas pueden suministrar modelos implícitos para el funcionamiento familiar en la siguiente generación (Bowen, 1978, citado por López y Escudero, 2003), por lo cual es indispensable tener en cuenta la cultura familiar y los patrones reiterativos que se presentan en el embarazo adolescente, rescatando la función de la familia como el primer ente educador y el más importante en la formación de los hijos, y con mayor importancia en la salud sexual y reproductiva.

\section{REFERENCIAS BIBLIOGRÁFICAS}

Acción Social. Presidencia de la República (2010). Embarazo en Adolescentes, cuaderno 4(3). Bogotá, Colombia.

Aigaje, B. y Suárez, G. (2013). Respuesta familiar a embarazos en adolescentes menores de 19 años en la zona rural que acude al hospital "Pedro Vicente Maldonado" en el período comprendido entre noviembre del 2012 a abril 2013 (Tesis de Pregrado en Medicina). Pontificia Universidad Católica de Quito, Ecuador. Recuperada de: http://mx.123dok.com/document/8yd294gq-respuesta-familiar-a-embarazos-en-adolescentes-menores-a-19-anos-en-la-zona-rural-que-acude-al-hospital-pedro-vicente-maldonado-en-el-periodocomprendido-entre-noviembre-del-2012-a-abril-2013.html

Anderson, R. y Carter I. (1994). La conducta humana en el medio social. Enfoque Sistémico de la sociedad. Barcelona, España: Editorial Gedisa.

Castrillón, M. D. (2007). Discursos institucionales sobre la familia en Brasil y Colombia: ¿biologizar/nuclearizar, o reconocer su diversidad?. Revista Latinoamericana de Ciencias Sociales, Niñez y Juventud, 5, 83-124.

Consejo Nacional de Política Económica y Social (CONPES) (2012). Documento Conpes Social 147. Lineamientos para el desarrollo de una estrategia para la prevención del embarazo en la adolescencia y la promoción de proyectos de vida para los niños, niñas, adolescentes y jóvenes en edades entre 6 y 19 años. Bogota. Recuperado de https:// colaboracion.dnp.gov.co/CDT/Conpes/Social/147.pdf

Fernández, M. Soto, A (2017). Diseño de una estrategia de intervención 
psicosocial desde el enfoque de habilidades para la vida para la prevención del embarazo subsecuente en adolescentes (Tesis de maestría). Pontificia Universidad Javeriana, Santiago de Cali, Colombia.

Florez N., C. E. (2000). Las transformaciones sociodemográficas en Colombia durante el siglo XX. Bogotá, Colombia: Banco de la República-Tercer Mundo Editores. Recuperado de https://www.researchgate. net/publication/283016431_Determinantes_Proximos_de_la_Fecundidad_en_Colombia_analisis_regional_2005

Fondo de Población de las naciones Unidas (2013). Informe estado de la población mundial 2013: Maternidad en la niñez. Enfrentar el reto del embarazo en adolescentes. Nueva York.

Gallo, N. y Molina, A. (2008). Evaluación de impacto proyecto salud sexual y reproductiva de adolescentes en Medellín, "Sol y Luna" componente cualitativo, 2008. Revista Salud Pública Medellín, Alcaldía de Medellín.

García, Y. (2008). Familias en Colombia. Transiciones hacia el siglo XXI. Ediciones Universidad Simón Bolívar. Barranquilla, Colombia: Editorial Mejoras Ltda.

Gracia, E. y Musitu, G. (2000). Psicología Social de la Familia. Buenos Aires, Argentina: Editorial Paidós.

González A, E, Leal F, I, Molina G, T y Chacón C, P. (2013). Patrón intergeneracional del embarazo adolescente en las hijas de una cohorte de mujeres que controlaron su primer embarazo en un centro integral para adolescentes embarazadas. Revista chilena de obstetricia y ginecología, 78(4), 282-289. https://dx.doi.org/10.4067/S0717-75262013000400006

Jones D. (2010). Diálogos entre padres y adolescentes sobre sexualidad: discursos morales y médicos en la reproducción de las desigualdades de género. Interface-Comunicação, Saúde, Educação. 14(32), 171-182. Recuperado dehttp://www.scielo.br/scielo. php?script=sci_arttextypid=S1414-32832010000100014

Komura, L. (2008). Maternidade na adolescencia em uma comunidade de Baixa Renda: experiencias reveladas pela historia oral. Revista Latinoamericana de Emfermagen, 16(2), 1-8.

López, S. y Escudero, V. (2003). Familia, Evaluación e Intervención. 
Madrid, España: Editorial CCS.

Magallanes D.E.A., Albornoz A.N., Mazuera, A.R., Machado M.J.E., Ramírez L., R.H., Vivas F., C.Z., Aleta A., J.D. (2015). El Táchira en cifras 2014. Maternidad y Embarazo en adolescentes. San Cristóbal, Venezuela: Observatorio Social, Universidad Católica del Táchira. Recuperado de: www.ucat.edu.ve/oset

Maturana, H. y Varela, F. (1998). De máquinas y seres vivos. Autopoiesis: la organización de lo vivo. Chile: Editorial Universitaria.

Mazuera Arias, R., Albornoz Arias, N.C., Ramírez Martínez, C., Carreño Paredes, M.T., Peinado Contreras, Y.C., Morffe Peraza, M.A., Gallardo Pérez, H.J. (2017). Maternidad adolescente en el Norte de Santander. Maracaibo, Venezuela: Universidad del Zulia.

Ministerio de Salud y Protección Social (2014). Protocolo de atención de la menor de 15 años embarazada. Recuperado de https://www.minsalud.gov.co/sites/rid/Lists/BibliotecaDigital/RIDE/VS/PP/SM-Protocolo-atencion-embarazada-menor-15.pdf

Mottrie, C., Coster, L., y Duret, I. (2006). Devenir mère: transformations des liens et des lieux familiaux lors des grossesses survenant à l'adolescence. Cahiers critiques de thérapie familiale et de pratiques de réseaux, 37(2), 121-137.

OMS (2011). El embarazo en la adolescencia. Organización Mundial de la Salud. Ginebra: OMS.

Narez, M. (2009). Influencia de la dinámica familiar en la presencia de conductas de riesgo en adolescentes del Instituto Manuel C. Silva en Villa de Álvarez, Colima. (Tesis de Licenciatura). México. Recuperada de: http:// digeset.ucol.mx/tesis_posgrado/Pdf/NARES_REYES_MARIA.pdf

Nieto, J. Rincón, L. Ávila, Y. Mariño, L., y Forero, M. (2011). Aproximación a los significados de paternidad, maternidad y embarazo adolescente en contextos de desplazamiento. Editor. En Embarazo adolescente en Bogotá: construir nuevos sentidos y posibilidades para el ejercicio de derechos. Fondo de Población de las Naciones Unidas. Bogotá. pp. 57-106.

Noblega, M. (2009). La maternidad en la vida de las adolescentes: Implicancias para la acción. Revista de Psicología, 1, 29-54. 
Noguera, N., y Alvarado, H. (2011). Embarazo en adolescentes: una mirada desde el cuidado de enfermería. Revista Colombiana de Enfermería, 7(7), 151-160. Recuperado de: http://www.uelbosque.edu.co/ sites/default/files/publicaciones/revistas/revista_colombiana_enfermeria/volumen7/embarazo.pdf

Piñeros, M. (2014). Significados asociados a la maternidad y paternidad en adolescentes de la ciudad de Cali (Tesis de pregrado inédita). Pontificia Universidad Javeriana, Santiago de Cali, Colombia.

Profamilia(2015).EncuestaNacionaldeDemografíaySaludENDS2015.Colombia. Recuperado de: http://www.profamilia.org.co/encuentas/Profamilia/ Profamilia/index.php?option $=$ comcontentyview $=$ articleyid $=62$ yltemid $=9$

Rojas Betancur, M., Méndez Villamizar, R., Álvarez Nieto, C. (2016). El papel de la familia en la normalización del embarazo a temprana edad. Universidad Autónoma del Caribe. Revista Encuentros, 14(01), 139-150. Roizblatt, A. (2009). Terapia Familiar y de Pareja. Chile: Editorial Mediterráneo Ltda.

Salinas, S., Castro, M., y Fernández, C. (2014). Vivencias y relatos sobre el embarazo en adolescentes. Una aproximación a los factores culturales, sociales y emocionales a partir de un estudio en seis países de la región, informe final. UNICEF.

Sevilla, T. M., y Orcasita, L. T. (2014). "Hablando de sexualidad": una mirada de los padres y las madres a los procesos de formación con sus hijos/as adolescentes en estratos populares de Cali. Avances en Enfermería, 32, 191-205.

UNICEF (2014). Vivencias y relatos sobre el embarazo en adolescentes. Recuperado de: https://www.unicef.org/lac/UNICEF_PLAN_embarazo_adolescente_2015.pdf

Xitlali, M. y Rivas, M. (2011). Percepcion de la familia enfermedad crónica. Revista Electrónica de Psicología Iztacala,14(4), 268-94

Cómo citar este capítulo:

Arenas-Villamizar, V.V., Fernández-Delgado, M.K., y Martínez-Santana, M.C. (2017). Cultura familiar y patrones reiterativos en salud sexual y reproductiva de madres adolescentes en la frontera Colombovenezolana (departamento Norte de Santander y Táchira). En Albornoz-Arias, N., Mazuera-Arias, R., Espinosa-Castro, J.F. (Ed.), Adolescencia: su relación con la familia, educación y sexualidad. Un enfoque transdisciplinario (pp.169-206). Barranquilla, Colombia: Ediciones Universidad Simón Bolívar. 